\title{
In What Sense is a Low-Reynolds Mixing Layer Stable?
}

\author{
BHATTACHARYA. P., GOVINDARAJAN. R., NARASIMHA. R. \\ Jawaharlal Nehru Centre for Advanced Scientific Research, Bangalore
}

\section{Introduction}

The Orr-Sommerfeld equation governs the evolution of linear disturbance modes in a strictly parallel flow. For the plane incompressible mixing layer this equation yields a critical Reynolds number equal to zero (Betchov \& Szewczyk 1963). This result has been difficult to understand for a long time, for 'energy' arguments show that there exists a nonzero Reynolds number, however small, below which viscosity would damp out any disturbance. It has often been suggested (e.g. Drazin \& Reid, 2004) that the resolution of this paradox is possible only by considering the non-parallelism of the mixing layer. As the rate of growth of the mixing layer and hence also is infinite at zero Reynolds, hence also at the critical Reynolds number predicted, and the degree of non-parallelism of the flow increases at lower Reynolds numbers, the Orr-Sommerfeld result is derived making an assumption that is grossly inadequate.

Recently, Bhattacharya et al. (2006) - henceforth referred to as I - used a nonparallel analysis to show that the critical Reynolds number $\operatorname{Re}_{\mathrm{cr}}$ for the mixing layer is not zero. When expressed with the velocity difference $\Delta$ and vorticity thickness $\delta_{\infty}$ as velocity and length scales respectively, they found that $\mathrm{Re}_{\mathrm{cr}}$ is about 30. The analysis therein follows the minimal composite theory developed in Govindarajan \& Narasimha (1997, 2001,2005 ) chiefly for boundary layers. As is well-known, the marginal stability curve in non-parallel theory depends on a number of factors: the precise disturbance quantity that is being monitored, the choice of trajectory along which the quantity is monitored, etc. To avoid this complexity the quantity used in I to define $\operatorname{Re}_{\mathrm{cr}}$ was an integral of the disturbance kinetic energy over a similarity coordinate $y$, i.e. a function only of the streamwise distance $x$.

In the present work, we propose to discuss the spatial evolution of the disturbance field. Results on three definitions of growth-rate were given by Gaster (1974, (a)-(c), p. 472 and Figures 2,3) in his non-parallel stability analysis of the boundary-layer. Although the differences in the stability curves were not entirely negligible, there was a lower-bound in $R_{\mathrm{cr}}$ of about 500 . In the present case, where the critical Reynolds number is much lower, we show that alternative definitions for the growth-rate give rise to qualitatively different stability loops of the mixing layer. Care must therefore be exercised when interpretations are offered regarding the physical processes underlying the instability of the mixing layer.

We investigate here the physical behaviour of the disturbance kinetic energy in order to gain insight into the character of the stability of the flow. 


\section{Problem Statement}

The mean flow in the incompressible mixing layer (see Figure 1 for notation) we consider possesses a similarity solution in terms of a similarity function $\phi(y)$, related to the dimensional streamfunction

$$
\Phi_{d}\left(x_{d} y_{d}\right)=\frac{2 \Lambda}{1+\Lambda} U_{\infty} \delta_{\omega}\left(x_{d}\right) \Phi(y), \quad \Phi^{\prime}(y)=U / U_{\infty} .
$$

where $\Delta=\left(\mathrm{U}_{\infty}-\mathrm{U}_{-\infty}\right) /\left(\mathrm{U}_{\infty}+\mathrm{U}_{-\infty}\right)$ is the velocity ratio parameter.

As a first step we simulate the flow as it might be observed in a wave-maker experiment. To this end a single disturbance mode, of dimensional frequency $\omega_{d}$, is introduced into the flow at an appropriate station. Since the temporal variation of the dimensional kinetic energy of the disturbance motion $k_{d}$ is purely sinusoidal, it is sufficient to analyse its average over one time period.

Following the minimal composite theory applied to the mixing layer as in I, the amplitude $\phi$ of the disturbance streamfunction is decomposed into a lowest-order solution $\phi_{m}$ and a higher-order correction $\phi_{h}$, that is

$$
\phi(x, y)=A(x) \phi_{m}(x, y)+\epsilon \phi_{h}(x, y)
$$

where

$$
\hat{\phi}_{d}=\left(\delta_{\omega} \Delta\right) \phi(x, y) \exp \left[\mathrm{i}\left(\int \alpha \mathrm{d} x-\omega_{d} t_{d}\right)\right]
$$

is the dimensional streamfunction corresponding to the mode $\omega_{d}$. Further, $A=A(x)$ and $\alpha=$ $\alpha(x)=\alpha_{r}+i \alpha_{i}$ represent the amplitude function and the non-dimensional wavenumber of the disturbance respectively. The analysis in I shows that the higher-order correction $\varepsilon \phi_{h}$ in (1) is $O\left(R^{-2 / 3}\right)$ compared to $A \phi_{m}$. The non-dimensionalisation adopted here is identical to that in I, namely

$$
\mathrm{d} x_{d}=\delta_{\omega} \mathrm{d} x, \quad y_{d}=\delta_{\omega} y \quad \text { and } \quad R \equiv \delta_{\omega} \Delta / \nu
$$

Note that $y$ is (by definition) the similarity coordinate for the mean flow in the mixing layer.

Now the local kinetic energy density of the disturbance is 


$$
k_{d} \equiv \frac{1}{2}\left(u_{d}^{\prime 2}+v_{d}^{\prime 2}\right)
$$

where

$$
u_{d}^{\prime}=\left(\frac{\partial \hat{\phi}_{d}}{\partial y_{d}}\right)_{r} \text { and } v_{d}^{\prime}=\left(\frac{\partial \widehat{\phi}_{d}}{\partial x_{d}}\right)_{r}
$$

subscript $\mathbf{r}$ standing for 'real part of'.

Substituting for the $\mathrm{r}$ ) city components using (1): $\mathrm{r}:,{ }^{\prime}$ then write the spatial distribution of the disturban nergy in the form

$$
\left\langle k_{d}\right\rangle\left(x_{d}, y_{d}\right) \equiv \frac{1}{4} \Delta^{2}\left(k_{0}+k_{\epsilon}+k_{R}\right) \exp \left(-2 \int_{x_{0}}^{x} \alpha_{i} \mathrm{~d} x\right)+o\left(R^{-1}\right),
$$

where

$$
\begin{aligned}
k_{0} & =\left|A D \phi_{m}\right|^{2}+\left|\alpha A \phi_{m}\right|^{2}, \\
k_{\epsilon} & =2 \epsilon\left(A D \phi_{m} D \phi_{h}^{*}+|\alpha|^{2} A \phi_{m} \phi_{h}^{*}\right)_{\mathrm{r}} \\
k_{R} & =2|A|^{2}\left(\mathrm{i} \alpha \phi_{m}\left[\frac{p}{R}+\frac{A^{\prime *}}{A^{*}}+\partial_{x}-\frac{1}{R} p y D\right] \phi_{m}^{*}\right),
\end{aligned}
$$

and the angular brackets \langle\rangle denote the average over one time period $2 \pi / \omega_{d}$. In these expressions the asterisk denotes the complex-conjugate, the prime on $A$ denotes a derivative taken with respect to $x$, and $\mathrm{D}=\partial / \partial \mathrm{y}$. Note that $k_{0}, k_{\varepsilon}$ and $k_{R}$ are contributions to the disturbance kinetic energy at $O(1), O(\varepsilon)$ and $O\left(R^{-1}\right)$ levels respectively. Fixing the amplitude level of the disturbance to be $A_{0}$ at the location where it is introduced, say at $x_{d}=$ $x_{d 0}$, we further define

$$
\kappa \equiv \frac{\left\langle k_{d}\right\rangle}{\left|A_{0}\right|^{2} \Delta^{2}},
$$

as the non-dimensional disturbance kinetic energy. Two integral quantities are also defined as follows:

$$
K(x)=\int_{-\infty}^{\infty} \kappa \mathrm{d} y
$$




$$
\bar{K}(x)=\int_{-\infty}^{\infty} \kappa \mathrm{d}\left(\frac{y_{d} \Delta}{\nu}\right)=R(x) K(x)
$$

Both these integrals are non-dimensional, but while $K$ is an integral over the similarity variable $y, \bar{K}$ uses the length-scale $(v / \Delta)$ which is independent of $x$, unlike $\delta_{\omega}$ increases with the streamwise distance like $x_{d / 2}^{1 / 2}$. Thus

$$
\mathrm{d} \delta_{\omega} / \mathrm{d} x_{d}=p / R
$$

where $p$ is a constant depending on the velocity ratio parameter $\Lambda=\Delta /\left(2 U_{\infty}-\Delta\right)$ and is given by

$$
p=\left(\frac{\Lambda}{1+\Lambda}\right)\left(\frac{|2 \Lambda /(1+\Lambda)|}{\max \left|\Phi^{\prime \prime}\right|}\right)^{2},
$$

where $\Phi^{\prime}(y)=U_{d}\left(y_{d}\right) / \Delta$ is the derivative in $y$ of the similarity streamfunction $\Phi(y)$ used to specify the mean-flow, $U_{\infty}$ is the free-stream velocity of the faster stream and the prime on $\Phi$ denotes a derivative with respect to $y$. Equation (10) implies that the rate of growth of $\bar{K}$ in the downstream direction includes the effect of the spreading of the mixing layer, or

$$
\frac{1}{\bar{K}} \frac{\mathrm{d} \bar{K}}{\mathrm{~d} x}=\frac{1}{K} \frac{\mathrm{d} K}{\mathrm{~d} x}+\frac{p}{R}
$$

In I the growth-rate $g$ corresponds to the quantity $K$ above. Using (4)-(6) and (7)-(9) it is straightforward to show that the spatial growth-rates of $\kappa$ and $K$ are given by

$$
\begin{aligned}
\frac{1}{\kappa} \frac{\partial \kappa}{\partial x}= & -2 \alpha_{i}+2\left(\frac{A^{\prime}}{A}\right)_{r} \\
& +\frac{2\left(\partial_{x} D \phi_{m} D \phi_{m}^{*}+\left|\phi_{m}\right|^{2} \alpha^{*} \alpha^{\prime}+|\alpha|^{2} \phi_{m}^{*} \partial_{x} \phi_{m}\right)_{r}}{\left|D \phi_{m}\right|^{2}+\left|\alpha \phi_{m}\right|^{2}} \\
& +o\left(R^{-1}\right), \\
\frac{1}{K} \frac{\mathrm{d} K}{\mathrm{~d} x}= & -2 \alpha_{i}+2\left(\frac{A^{\prime}}{A}\right)_{r} \\
& +\frac{\int_{-\infty}^{\infty} 2\left(\partial_{x} \phi_{m} D \phi_{m}^{*}+\left|\phi_{m}\right|^{2} \alpha^{*} \alpha^{\prime}+|\alpha|^{2} \phi_{m}^{*} \partial_{x} \phi_{m}\right)_{r} \mathrm{~d} y}{\int_{-\infty}^{\infty}\left(\left|D \phi_{m}\right|^{2}+\left|\alpha \phi_{m}\right|^{2}\right) \mathrm{d} y} \\
& +o\left(R^{-1}\right) ; \quad
\end{aligned}
$$


and the growth-rate of $\bar{K}$ is obtained by using (13) in (11).

The determine the evolution of $\kappa$ we start with the equation for the disturbance energy in two dimensional flow, obtained by taking the scalar product of the disturbance momentum equation with the disturbance velocity. We then obtain (e.g. from Schlichting \& Gersten, 2004, pp. 503-4),

$$
\begin{aligned}
& \rho\left(U \frac{\partial\left\langle k_{d}\right\rangle}{\partial x_{d}}+V \frac{\partial\left\langle k_{d}\right\rangle}{\partial y_{d}}\right)=-\left\langle u_{d}^{\prime} \frac{\partial \hat{p}_{d}}{\partial x_{d}}+v_{d}^{\prime} \frac{\partial \hat{p}_{d}}{\partial y_{d}}\right\rangle \\
& \text { advection } \\
& \text { pressure } \\
& \text { transport } \\
& +\rho v\left[\frac{\partial^{2}}{\partial x_{d}^{2}}\left(\left\langle k_{d}\right\rangle+\left\langle u_{d}^{\prime 2}\right\rangle\right)+2 \frac{\partial^{2}}{\partial x_{d} \partial y_{d}}\left\langle u_{d}^{\prime} v_{d}^{\prime}\right\rangle+\frac{\partial^{2}}{\partial y_{d}^{2}}\left(\left\langle k_{d}\right\rangle+\left\langle v_{d}^{\prime 2}\right\rangle\right)\right] \begin{array}{l}
\text { viscous } \\
\text { diffusion }
\end{array} \\
& -\rho\left(\left\langle u_{d}^{\prime 2}\right\rangle \frac{\partial U}{\partial x_{d}}+\left\langle u_{d}^{\prime} v_{d}^{\prime}\right\rangle \frac{\partial V}{\partial x_{d}}+\left\langle u_{d}^{\prime} v_{d}^{\prime}\right\rangle \frac{\partial U}{\partial y_{d}}+\left\langle v_{d}^{\prime 2}\right\rangle \frac{\partial V}{\partial y_{d}}\right) \quad \begin{array}{l}
\text { turbulent } \\
\text { production }
\end{array} \\
& -\rho v\left[2\left\langle\left(\frac{\partial u_{d}^{\prime}}{\partial x_{d}}\right)^{2}+2\left(\frac{\partial v_{d}^{\prime}}{\partial y_{d}}\right)^{2}\right\rangle+\left\langle\left(\frac{\partial u_{d}^{\prime}}{\partial y_{d}}\right)^{2}+2\left(\frac{\partial v_{d}^{\prime}}{\partial x_{d}}\right)^{2}\right\rangle\right] \quad \text { dissipation. }
\end{aligned}
$$

The differences between the above expressions and those in (16.14)-(16.19) in Schlichting \& Gersten (2004), are as follows:

1. The equations above are two-dimensional, $\partial / \partial z W, w_{d}^{\prime}$ are identically zero everywhere in the flow.

2. The contribution to turbulent diffusion from the advection of disturbance energy, by the disturbance field itself, is neglected because it is of a higher-order (in disturbance magnitude units) compared to the other terms in the equation. Also note that the disturbance pressure is of a lower order than the disturbance velocity field.

To derive the expression for the pressure transport term, we write the dimensional disturbance pressure field as a waveform

$$
\hat{p}_{d}=\rho \Delta^{2} \Pi(x, y) \exp \left\lfloor\left(\int \alpha d x-\omega_{d} t_{d}\right)\right] .
$$

The pressure gradient in both $x$ and $y$ directions can be computed using the momentum equations, written in vector form as 


$$
\bar{\nabla}_{d} \hat{p}_{d}=\frac{\partial \bar{u}_{d}}{\partial t_{d}}-\left(\bar{U} \cdot \bar{\nabla}_{d}\right) \bar{u}_{d}-\left(\bar{u}_{d} \cdot \bar{\nabla}_{d}\right) \bar{U}+\frac{1}{R} \bar{\nabla}_{d}^{2} \bar{u}_{d}
$$

where $\bar{\nabla}_{d}=\left\{\partial / \partial x_{d}, \partial / \partial y_{d}\right\}, \bar{U}=\{U, V\}$ and $\bar{u}_{d}=\left\{u_{d}^{\prime}, v_{d}^{\prime}\right\}$. By substituting for the velocity components using (1) and (2), and similarly for the mean flow and disturbance pressure, followed by expanding and retaining terms only upto $O\left(\mathrm{R}^{-1}\right)$, we get the normal and streamwise directions respectively

$$
\begin{aligned}
D \Pi= & \frac{-\alpha^{2}\left(\Phi^{\prime}-c\right) A \phi_{m}}{O(1)}-\frac{\alpha^{2}\left(\Phi^{\prime}-c\right) \varepsilon \phi_{h}}{O(\varepsilon)}+i \Phi^{\prime} \partial_{x}\left(A \alpha \phi_{m}\right) \\
& +i \alpha A\left(\Phi^{\prime}-c\right)\left[\frac{p}{R}+\frac{A^{\prime}}{A}+\partial_{x}-\frac{p y}{R} D\right] \phi_{m} \\
& -\frac{1}{R} i \alpha A p \Phi D \phi_{m}+\frac{1}{R} i \alpha A p y \Phi^{\prime \prime} \phi_{m} \\
& -\frac{1}{R} i \alpha A\left(D^{2}-\alpha^{2}\right) \phi_{m}, \\
\left(i \alpha+\partial_{x}-\frac{p y}{R} D\right) \Pi= & \frac{-i \alpha\left[\left(\Phi^{\prime}-c\right) A D \phi_{m}-\Phi^{\prime \prime} A \phi_{m}\right]}{O(1)} \\
& \frac{-i \alpha\left[\left(\Phi^{\prime}-c\right) \varepsilon D \phi_{h}-\Phi^{\prime \prime} \varepsilon \phi_{h}\right]}{O(\varepsilon)} \\
& +\left[\begin{array}{l}
\frac{1}{R} p \Phi A D^{2}-A \Phi^{\prime}\left(\partial_{x}+\frac{A^{\prime}}{A}\right) D+\frac{1}{R} p A \Phi^{\prime \prime} \\
\left.+A \Phi^{\prime \prime}\left(\partial_{x}+\frac{A^{\prime}}{A}\right)+\frac{1}{R} p A D^{3}-\frac{1}{R} A \alpha^{2} D\right] \phi_{m}
\end{array}\right.
\end{aligned}
$$

where $c(=\omega / \alpha)$ is the non-dimensional phase speed of the wave. Using these in the expression for pressure-transport and averaging over one time-period we obtain the expression for $T_{p}$ given in (18) below. While averaging it should be noted that $\partial_{x} \omega=\omega p / R$, since $\omega_{d}$ (not $\omega$ ) is invariant in $x$. (In (16), and in the sequel, $O(1)$ terms appear in blue, $O(\varepsilon)$ terms appear in red and $O\left(R^{-1}\right)$ terms appear in black.) Using these in (14), and again keeping terms upto $O\left(R^{-1}\right)$, we can write 


$$
\Phi^{\prime} \frac{\partial \kappa}{\partial x}=P-\varepsilon+D_{p}+T+D_{\nu}
$$

where

$$
\begin{aligned}
& P=-\frac{1}{2} \Phi^{\prime \prime}|A|^{2}\left(D \phi_{m} \mathrm{i} \alpha^{*} \phi_{m}^{*}\right)_{r}-\frac{1}{2} \epsilon \Phi^{\prime \prime}\left(\mathrm{i} \alpha^{*} A D \phi_{m} \phi_{h}^{*}+\mathrm{i} \alpha A \phi_{m} D \phi_{h}^{*}\right)_{r} \\
& -\frac{1}{2} \Phi^{\prime \prime} \frac{p y}{R}\left|A \alpha \phi_{m}\right|^{2}+\frac{1}{2} \Phi^{\prime \prime}|A|^{2}\left\{D \phi_{m}^{*}\left(\partial_{x}+\frac{A^{\prime}}{A}+\frac{p}{R}\right) \phi_{m}\right\}_{r}, \\
& \varepsilon=\frac{1}{2 R}\left\{4\left|\alpha D \phi_{m}\right|^{2}+\left|D^{2} \phi_{m}\right|^{2}+\left|\alpha^{2} \phi_{m}\right|^{2}+2\left(\alpha^{* 2} \phi_{m}^{*} D^{2} \phi_{m}\right)_{r}\right\}, \\
& T_{p}=-\frac{1}{2} \alpha_{i} \Phi^{\prime}\left(\left|A D \phi_{m}\right|^{2}+\left|\alpha A \phi_{m}\right|^{2}\right)-\frac{1}{2} \Phi^{\prime \prime}|A|^{2}\left(\mathrm{i} \alpha \phi_{m} D \phi_{m}^{*}\right)_{r} \\
& -\frac{1}{2} \epsilon\left[2 \alpha_{i} \Phi^{\prime}|\alpha|^{2}\left\{A \phi_{m} \phi_{h}^{*}\right\}_{r}+2 \alpha_{i} \Phi^{\prime}\left\{A D \phi_{m} D \phi_{h}^{*}\right\}_{r}\right. \\
& \left.+\Phi^{\prime \prime}\left(\mathrm{i} \alpha A^{*} D \phi_{m}^{*} \phi_{h}\right)_{r}+\Phi^{\prime \prime}\left(\mathrm{i} \alpha A \phi_{m} D \phi_{h}^{*}\right)_{r}\right] \\
& -\frac{1}{2 R}|A \alpha|^{2}\left(\phi_{m}^{*} D^{2} \phi_{m}\right)_{r}-\frac{1}{2 R}|A|^{2}\left(D \phi_{m}^{*} D^{3} \phi_{m}\right)_{r} \\
& +\frac{1}{2 R}\left(\alpha^{2}\right)_{r}\left[\left|A D \phi_{m}\right|^{2}+\left|\alpha A \phi_{m}\right|^{2}\right] \\
& -\frac{1}{2 R} p \Phi|A|^{2}\left(D \phi_{m}^{*} D^{2} \phi_{m}\right)_{r}-\frac{1}{2 R} p \Phi|\alpha A|^{2}\left(\phi_{m}^{*} D \phi_{m}\right)_{r} \\
& +\frac{1}{2 R} p y \Phi^{\prime \prime}\left|\alpha A \phi_{m}\right|^{2}-\frac{1}{2} \Phi^{\prime \prime}|A|^{2}\left(D \phi_{m}^{*}\left\{\frac{p}{R}+\partial_{x}+\frac{A^{\prime}}{A}\right\} \phi_{m}\right)_{r} \\
& +\Phi^{\prime} \alpha_{i}^{2} \frac{p}{R}\left|A \phi_{m}\right|^{2}+\frac{1}{2} \Phi^{\prime}|\alpha A|^{2}\left(\phi_{m}^{*} \partial_{x} \phi_{m}\right)_{r}+\frac{1}{2} \Phi^{\prime}\left|A \phi_{m}\right|^{2}\left(\alpha^{*} \alpha^{\prime}\right)_{r} \\
& +\frac{1}{2} \Phi^{\prime}|A|^{2}\left(D \phi_{m}^{*}\left\{\partial_{x}+\frac{A^{\prime}}{A}\right\} D \phi_{m}\right)_{r}+\frac{1}{2} \Phi^{\prime}\left|\alpha A \phi_{m}\right|^{2}\left(\frac{A^{\prime}}{A}\right)_{r} \\
& -\Phi^{\prime} \alpha_{i}|A|^{2}\left(\operatorname{i} \alpha \phi_{m}\left\{\partial_{x}-\frac{p y}{R} D+\frac{A^{\prime *}}{A^{*}}\right\} \phi_{m}^{*}\right)_{r}, \\
& T_{V}=\frac{2 p \Phi}{R}\left[\left(D \phi_{m} D^{2} \phi_{m}^{*}\right)_{r}+|\alpha|^{2}\left(D \phi_{m} \phi_{m}^{*}\right)_{r}\right] \\
& \text { and } \quad T_{v}=\frac{1}{2 R}\left\{\left|D^{2} \phi_{m}\right|^{2}+\left|\alpha^{2} \phi_{m}\right|^{2}+4\left|\alpha D \phi_{m}\right|^{2}+2\left(\alpha^{* 2} \phi_{m}^{*} D^{2} \phi_{m}\right)_{r}\right. \\
& -\left(\alpha^{2}\right)_{r}\left|\alpha \phi_{m}\right|^{2}-\left(\alpha^{2}\right)_{r}\left|D \phi_{m}\right|^{2}+|\alpha|^{2}\left(\phi_{m}^{*} D^{2} \phi_{m}\right)_{r} \\
& \left.+\left(D \phi_{m} D^{3} \phi_{m}^{*}\right)_{r}\right\} \text {. }
\end{aligned}
$$

The terms here denote respectively the gain in disturbance kinetic energy due to shearproduction $(P)$, loss due to dissipation $(\varepsilon)$ and transport of disturbance kinetic energy rspectively by the disturbance pressure field $\left(\mathrm{T}_{p}\right)$, by the mean-flow in the normal direction $\left(T_{V}\right)$ and by viscous diffusion $\left(T_{v}\right)$. The only difference between (14) and (18) in the 
advection term in the former, and that labeled as $T_{v}$ in the latter. Following our choice of non-dimensionalization, the advection term in (14) can be expanded as

$$
\rho\left(\frac{U}{\delta_{\omega}} \frac{\partial\left\langle k_{d}\right\rangle}{\partial x}-\frac{U}{\delta_{\omega}} \frac{p y}{R \delta_{\omega}} D\left\langle k_{d}\right\rangle+V \frac{\partial\left\langle k_{d}\right\rangle}{\partial y}\right)
$$

The last two terms above are moved to the right hand side of the equation and appear as transport of the disturbance energy by the mean flow in the normal direction. Equation (12) is now retrieved by dividing the right hand side of (17) by the produce $\left(\Phi^{\prime} \kappa\right)$ and retaining terms upto $O\left(R^{-1}\right)$.

Note that the $O(\varepsilon)$ contributions to shear production $P$ and pressure transport $T_{p}$ depend on the higher order eigen-function $\varepsilon \phi_{h}$. This is obtained by using the amplitude evolution equation (I),

$$
\frac{\epsilon}{A} \mathcal{M} \phi_{h}=-\mathcal{H} \phi_{m}-\frac{A^{\prime}}{A} \mathcal{S} \phi_{m}
$$

where $\mathcal{M}, \mathscr{H}$ and $S$ are respectively . . The non-homogeneous linear equation (20) can be solved since $\varphi_{m}$ is known from the solution of the lowest-order equation

$$
\mathcal{M} \phi_{m}=0
$$

where $\mathcal{N}=\mathcal{M}+\mathcal{H}$ determines $\phi$ correct to $O\left(R^{-1}\right)$,

$$
\mathcal{N} \phi=o\left(R^{-1}\right)
$$

The numerical procedure for solving (20) yields a solution of the form $\phi_{\mathrm{hsol}}\left(=\mathrm{B} \phi_{\mathrm{m}}\right.$ $+\phi_{h}$ ), containing an arbitrary multiple $B$ of $\phi_{\mathrm{m}}$, where $B$ is a complex-valued constant. The multiple of $\phi_{\mathrm{m}}$ can be removed using a Gram-Schmidt orthogonalization

$$
\phi_{h}(y)=\phi_{h_{\mathrm{sol}}}(y)-\left(\frac{\int \phi_{h_{\mathrm{sol}}} \phi_{m}^{*} \mathrm{~d} y}{\int\left|\phi_{m}\right|^{2} \mathrm{~d} y}\right) \phi_{m}(y),
$$

the integrals being taken from $-\infty$ to $\infty$. 


\section{Analysis}

We now choose a value of the $x$-independent frequency parameter $v \omega_{d} \Delta^{-2}$ such that the corresponding mode is marginally growing near the critical Reynolds number reported in I. The eigenfunctions $\phi_{m}$ and $\phi_{h}$, and the wavenumber $\alpha$, are obtained at different streamwise locations. A rectangular domain $(x, y) \in\left[x_{0}, x_{1}\right] \times[-L, L]$ in non-dimensional space is considered. We use a forward-difference scheme to obtain $\kappa_{i+1}, j \equiv \kappa\left(x_{i+1}, y_{i}\right)$ from $\kappa_{i},{ }_{j}$ from the relation

$$
\kappa_{i+1, j}=\kappa_{i, j}\left\{1+\left(\frac{1}{\kappa} \frac{\mathrm{d} \kappa}{\mathrm{d} x}\right)_{i, j}\left(x_{i+1}-x_{i}\right)\right\}+o(1) .
$$

Similar formulations allow us to determine $K$ and $\bar{K}$.

While evaluating integrals, the contributions from the infinite tails are carefully included after verifying that the integrand has attained the asymptotic exponential decay at the boundary of the computational domain. The finite domain is taken to be sufficiently large so that this assumption holds. In the results reported the contribution from the tailregion contributed less than $10^{-3}$ percent to the total integral.

The analysis is carried out for three illustrative values of the velocity ratio parameter $\Lambda=49 / 50,1 / 3$ and $1 / 39$. In I we had noted that the critical Reynolds number expressed in the conventional scales $U_{\infty}$ and $l_{d}\left(=\sqrt{\left.x_{d} v / U_{\infty}\right)}\right.$, that is $R^{*}=U_{\infty} l_{d} / v$, is about 6 for $\Lambda=1$ and $\infty$ for $\Lambda=0$. Therefore the half-jet $(\Lambda=1 / 39)$ is close to the limit of shearless flow, which is stable at very high $R^{*}$.

For the half-jet a numerical difficulty was encountered while obtaining the growth rate $(1 / \kappa)(\partial \kappa / \partial x)$ for $y<<-1$, as division by the streamwise mean velocity $\Phi$ causes the growth-rate to diverge. [Theoretically $\kappa$ itself is very small in this region, making the product $\kappa \Phi^{\prime}$ finite. But computationally this was not obtained.] For this reason results are not reported in the region $y \ll-1$ for the half-jet case; instead we consider $\Lambda=49 / 50$ which is deemed sufficiently close to be representative of that extremity.

\section{Results}

The contours of $\kappa$ in the half-jet flow $\Lambda=49 / 50$ and nearly shearless flow $\Lambda=1 / 39$ are shown in Figures 2 and 3 for near-critical disturbance modes. To obtain this flow picture of the spatial distribution of disturbance energy the growth-rate was computed using (17), taking care to include only terms correct to $O\left(R^{-1}\right)$. The same quantity was also computed using the growth rate given by (12). The two computations were verified to be identical to six decimal places. 
It is apparent that upstream ( / downstream) of the streamwise location corresponding to $R \approx 39$ there is hardly any streamline in the core of the flow along which energy decays ( / grows) in the streamwise direction. Thus the picture is intuitively faithful to the concept of stability ( / instability). Before we fix this idea, we consider Figure 2 in some more detail.

The edges separating differently coloured regions correspond to trajectories in $\left(x_{d}-\right.$ $\left.y_{d}\right)$ space along which the mode is marginally stable. Consider now the isotachs (corresponding to constant $y$ values) that are superposed on this picture. The inflexion point is at $y=-0.11$. At every point in a neighbourhood of the inflexion point and upto a certain Reynolds number, the disturbance energy decays along an isotach; beyond the same Reynolds number it increases. The value of $R$ where the change occurs depends in general on position. In some regions, the disturbance energy starts amplifying at an earlier streamwise location compared to others. It will be seen that over a considerable portion of the mixing layer the energy decreases to a minimum and then starts increasing again along the streamlines. It is in this sense that we propose that it is appropriate to speak of the stability of the mixing layer below a Reynolds number of the order of 30 .

Figures 4 and 5 show the variation of the disturbance kinetic energy along selected streamlines. This variation confirms the conclusion drawn above. It is therefore interesting to see how the integrated effect, of the decaying nature of the disturbance in the core and the strong non-parallelism of the mixing layer, manifests itself. In I we had defined the quantity to be monitored as $K$, which owing to the integration over the similarity coordinate $y$, neglected the growing nature of the mixing layer. The thickness of the mixing layer is proportional to the vorticity-thickness and therefore its logarithmic rate of growth is also $p / R$. Hence, at low values of $R$ this quantity becomes progressively larger. Below we consider the integrated quantity $\bar{K}$ as defined in (9), the growth rate of which is given by (11).

For the same frequency $\omega_{d}$ considered above, the variation of the two integrated kinetic energies are shown in Figures 4 (corresponding to $\Lambda=49 / 50)$ and $7(\Lambda=1 / 39$ ) across the same range of $R$ as above. We see that $K$ decays upto $R \approx 30$ and then amplifies, but at relatively small rate. On the other hand $\bar{K}$ amplifies monotonically at all $R$. Note that a stretched scale is used to highlight the variation in $K$ in comparison to that of $\bar{K}$. Secondly the rate of growth of $\bar{K}$ is indistinguishable from $p / R$.

Note that in computing $\kappa$, the error made is $o(1)$, which is due to the integration over an $x$-domain that scales as $O(R)$. Therefore, the accuracy in predicting the point of instability by observing $K$ or $\bar{K}$ is also correct to that order. The Reynolds numbers at which the quantities $\kappa$ and $K$ start amplifying, in the contour and integral plots shown herein, must be interpreted with this in mind. In I, we had used the local growth rate $g$, which is obtained correct to $o(1 / R)$, to identify marginal stability. 


\section{Conclusion}

The present study puts into perspective what one understands by the stability of highly non-parallel flows. It is seen that the definition of growth rate, already known to be dependent on the choice of monitoring parameters, might give results that lead to qualitatively different interpretations regarding the nature of the stability of the flow. The mixing layer provides a striking example to illustrate this point. Thus it was found in the foregoing analysis that the integral of the disturbance energy over the physical coordinate $y_{d}$ grows continuously from very low Re, and hence points toward instability. On the other hand an integral taken over the similarity coordinate $y$, or the maximum $\kappa_{\max }$ in $y$ at any station $x$, shows stability upto $R \approx 30$. It is in this sense that the mixing layer has a non-zero critical Reynolds number. There is no inherent contradiction between these results in a strongly non-parallel flow, for one simple reason. The maximum disturbance kinetic energy density $\kappa_{\max }$ may drop substantially over a given streamwise extent of the flow, as shown in Figure 2, but its integral in $y_{d}$ may increase if the flow is rapidly thickening (see Figure 1 again). Thus the streamwise rate of change of the integral depends not only on the rate of change of the disturbance kinetic energy, but also on that of the local vorticity thickness. As $\bar{K} \propto R K$, it is possible that $K$ decays, even as $\bar{K}$ increases. The result that $\bar{K}$ grows at all $R$ seems to echo the parallel stability result of Betchov \& Szewczyk (1963). (Results from present computations for $R<10$ may not be accurate because the underlying minimal composite theory is based on large $R$ asymptotics.) However, we believe that such a conclusion is misleading because of two reasons. Firstly, within substantial regions of $x y$ space the disturbance energy decays continuously upto a significantly high Reynolds number. Secondly, it is the high degree of non-parallelism of the mean flow, given by the quantity $d \delta_{\omega} / d x_{d}=p / R$, that accounts for the growth of $\bar{K}$, which is explained by the spreading of a basically smaller disturbance over a rapidly growing $y$-domain.

We can state these results in slightly different terms. Define the $L_{p}$ norm of $\kappa$ by

Then we have

$$
\bar{K}_{p}=\left(\int_{-\infty}^{+\infty}|\mathrm{\kappa}| \bar{p} \mathrm{~d} Y\right)^{1 / \mathrm{p}}, \quad Y \equiv y_{d} \Delta /
$$

$$
\begin{aligned}
\bar{K}_{1} & =\bar{K} \\
\bar{K}_{\infty} & =\sup _{Y} \kappa .
\end{aligned}
$$

The results presented here show that while $\bar{K}_{\infty}$ exhibits a non-zero critical Reynolds number, $\bar{K}_{1}$ is consistent with a value of zero for $R_{c r}$. We cannot assert that $\bar{K}_{1}$ grows monotonically from $R=0$, because our calculations cannot be extended to extremely low values of $R$ : the minimal composite theory we use is not valid for small $R$. Nevertheless, it is a fact that $\bar{K}_{1}$ exhibits no minimum anywhere at least for $R>20$.

Thus, if $\bar{K}=\bar{K}_{1}$ is chosen as the norm, it points towards instability over the whole range of $R$ considered. On the other hand the integral $K$ over the similarity coordinate $y$ decays upto a value of $R$ in the range 28 to 39 depending on $\Lambda$, and hence possesses a critical $R$ in the same range. It is in this sense that the mixing layer has a non-zero critical 
Reynolds number. Thus, while the maximum disturbance kinetic energy density $\kappa_{\max }=\bar{K}_{\infty}$ drops substantially over a given streamwise extent of the flow (Figure 2), its integral in $y_{d}$ can increase if the flow is rapidly thickening (see Figure 4), as it does at low $R$.

Stability limits thus depend on how the norm is defined.

To conclude, the interpretation of the stability of highly non-parallel flows is not necessarily straightforward, and so it is revealing to analyse the disturbance energy evolution in different ways. Nevertheless, we wish to suggest that, from the point of view of predicting transition, the simple $\kappa_{\max }$ is likely to be the physically relevant norm.

\section{References}

Betchov R and Szewczyk A. 1963 Stability of a shear layer between parallel streams. Phys. Fluids 6:1391-1396.

Bhattacharya P, Manoharan M P, Govindarajan R and Narasimha R 2006 The critical Reynolds number of a laminar incompressible mixing layer from minimal composite theory J. Fluid Mech. 565:105-114.

Govindarajan R and Narasimha R 1997 A low-order theory for stability of non-parallel boundary layer flows. Proc. R. Soc. Lond. A 453:2537-2549.

Govindarajan R, Narasimha R 2001 Estimating amplitude ratio in boundary layer stability theory: a comparison between two approaches. J. Fluid Mech. 439:403-412.

Govindarajan R, Narasimha R 2005 Accurate estimate of disturbance amplitude variation from solution of minimal composite stability theory. Theor. Comput. Fluid Dyn. 19:229-235.

Schlichting H and Gersten K 2004 Boundary Layer Theory, 8th Ed. Springer. McGrawHill, New York. 


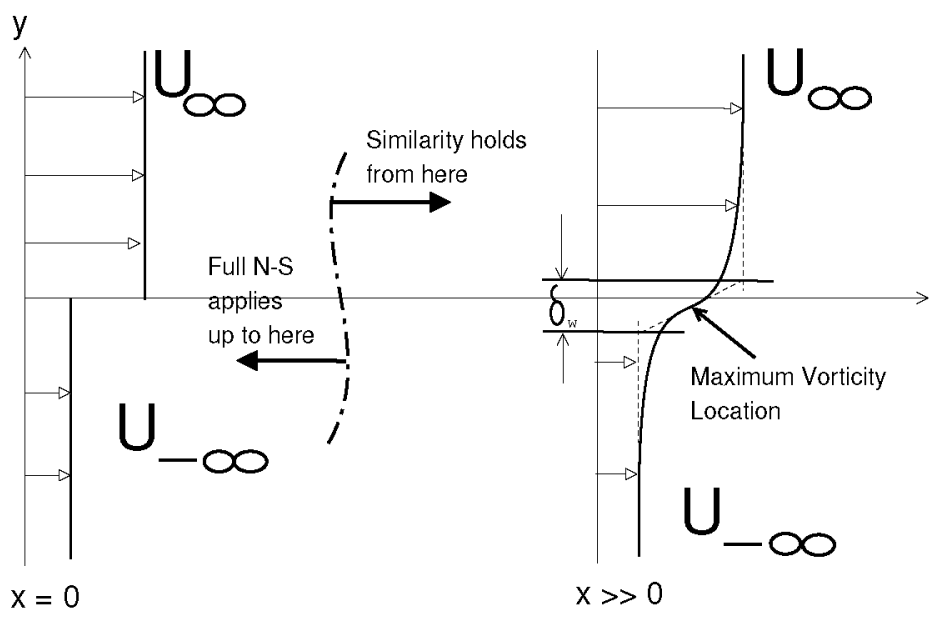

Figure 1: Geometry of the mean flow.



Figure 2: Spatial distribution of disturbance kinetic energy, half jet. 


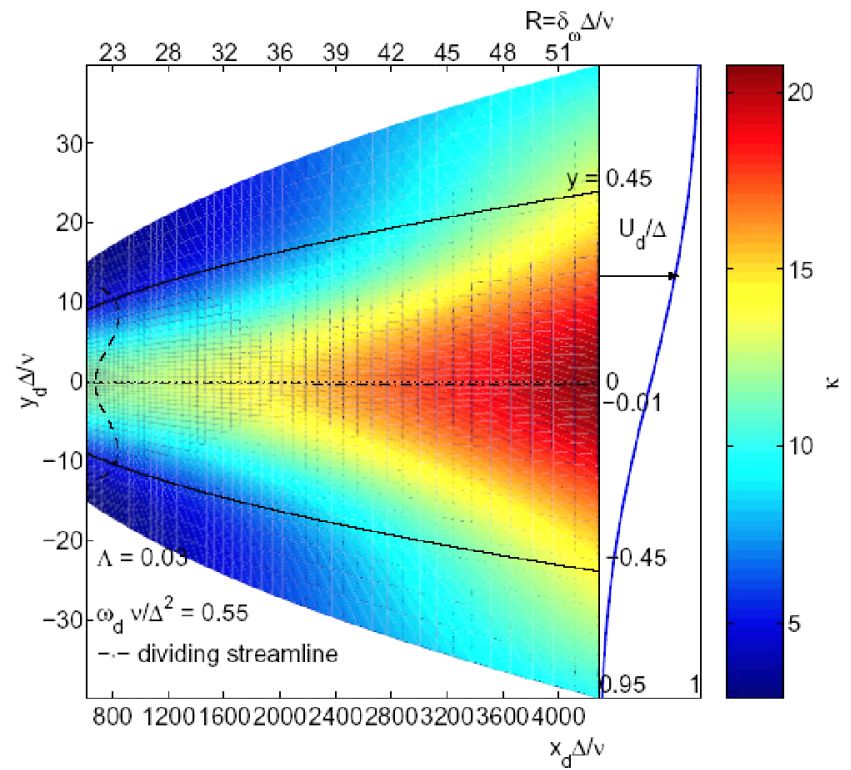

Figure 3: Spatial distribution of disturbance kinetic energy, nearly shear-less flow.

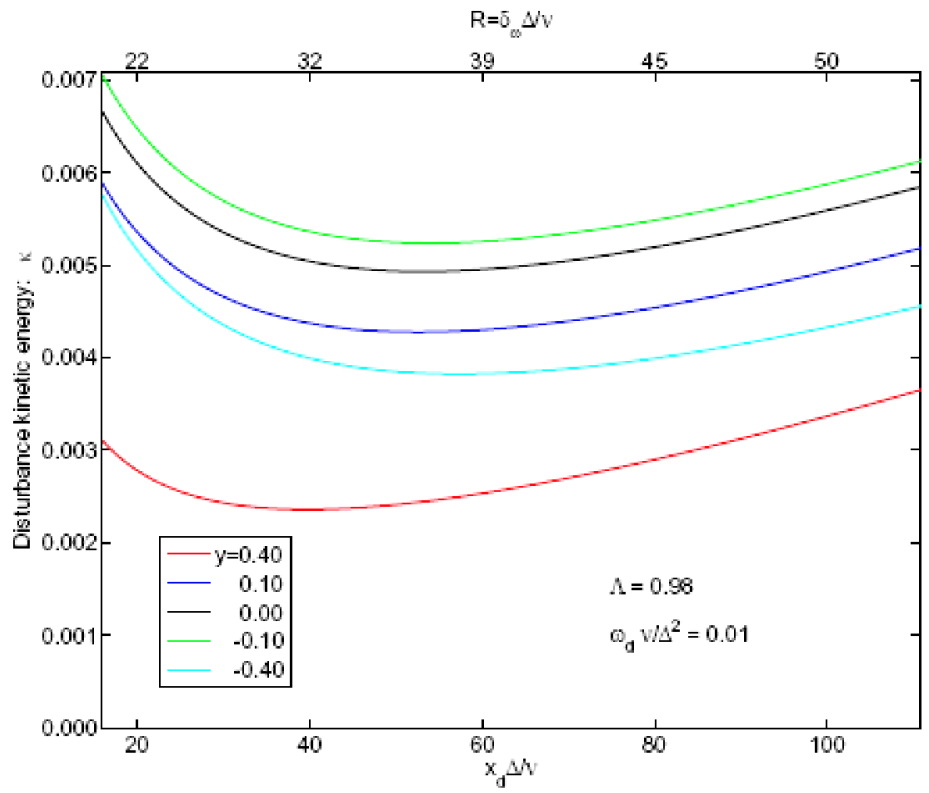

Figure 4: Kinetic energy density variation along isotachs in a near half-jet. 


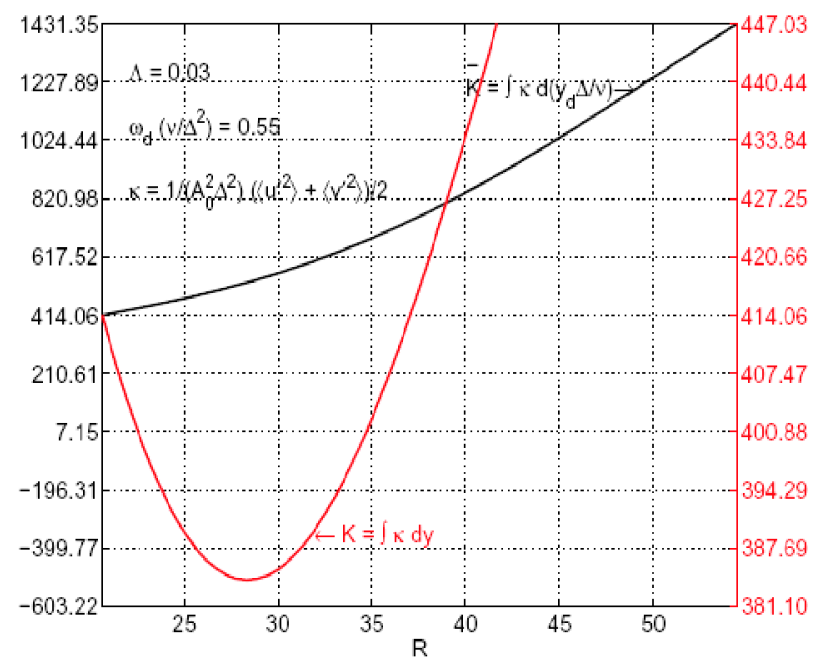

Figure 6: Comparison of the streamwise variation of $\bar{k}$ and $K$. near half-jet.

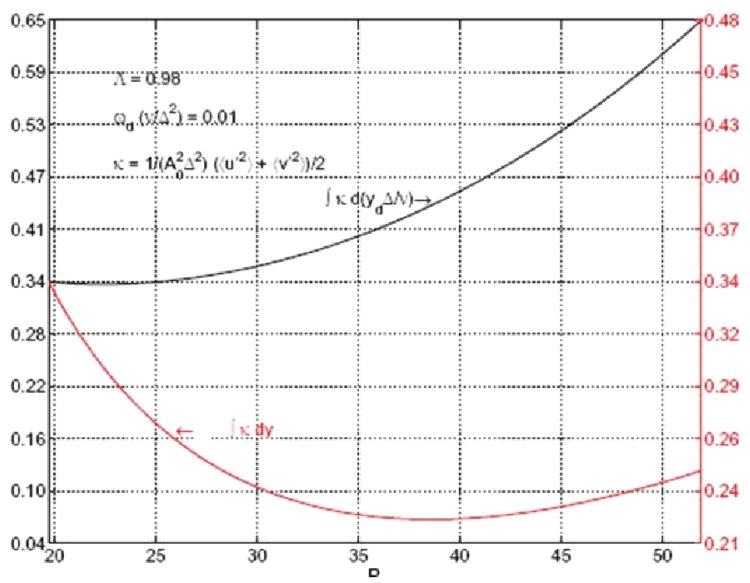

Figure 7: Comparison of the streamwise variation of $\bar{K}$ and $K$. nearly shear-less flow, legend on curves same as in Figure 6. 